\title{
Linear Precoding for the Downlink of Cognitive Radio Network
}

\author{
PENGPENG LAN, YANG XIAO, JINFENG KOU \\ Institute of Information Science, Beijing Jiaotong University \\ Beijing 100044,P.R. CHINA
}

\begin{abstract}
In this paper, a multiple-input-single-output (MISO) overlay cognitive radio (CR) network is considered in which a primary base station (PBS) and a cognitive base station (CBS) share the same spectrum to transmit the information. We consider a novel linear precoding technique to cannel the interferences which is bring about by the CBS's transmission. Three theorems about the design of the precoding and decoding matrices are put forward. With the linear precoding and decoding, the BER performance of the PBS and CBS are improved significantly. At the same time, the capacity of the PBS and CBS channels are analyzed.
\end{abstract}

Keywords: multiple-input-single-output(MISO);overlay; Cognitive radio (CR); precodin; decoding

Received: February 21, 2021. Revised: November 25, 2021. Accepted: December 16, 2021. Published: December 30, 2021.

\section{Introduction}

The use of cognitive radio (CR) has been recognized as a promising solution to improve the utilization efficiency of the current severely underutilized spectrum [1-3]. By allowing unlicensed secondary users (SUs) to coexist either opportunistically or concurrently with the licensed primary users (PUs), the shortage of wireless s pectrum resources is eased.

In the last decade, research on CR ha s focused on three main spectrum sharing paradigms: interweave underlay, and overlay [4]. Each of them requires a different level of cognition about the surrounding environment and a different level of sophistication which leads to different challenges. In interweave $\mathrm{CR}, \mathrm{CR}$ uses advanced spectrum-sensing techniques to detect spectrum holes (opportunities) in the PU's licensed band and transmits its own signals in the detected free bands. It must stop transmission as soon as the PU returns to the used bands so as not to cause any interference to the PU. In underlay $\mathrm{CR}$, the $\mathrm{CR}$ op erates under the constrain that the interference to the primary receiver must not exceed a given level [5]. On the other hand, the overlay CR can learn the primary message by overhearing primary ARQ transmissions and utilize knowledge of the m essage to perform spectrum overlay during retransmissions of the primary system[6]-[9]. As an alternative, [10] and [11] consider explicit cooperation of the primary and secondary systems.

In this paper, we consider a MISO overlay CR network where the PBS and CBS simu ltaneously transmit in the same spectrum to 2 PUs and SUs. We consider the communication scenario where PBS and CBS transmissions cause interference to each other. Such a scenario is justified in situations when the CBS is tran smitting in the se rvice area range of the PBS, thereby causing interference to each other [12]-[18].

As traditional in the overlay CR, it is assumed that the CBS has noncausal knowledge of the primary data vector [16-18].
This assumption is well documented in literature for th $\mathrm{e}$ overlay CR model [6]-[8]. In this paper, we develop a scheme that the PBS and the CBS use linear precoding to cancel the interference to PUs and SUs to $m$ ake PBS an $d$ the CBS sharing a same channel. At the same time, we have proposed three theorems about $t$ he construct of the precoding a nd decoding matrices. BER pe rformance and capacity of the channels are analyzed.

The rest of the paper is organized as follows. In Section II, we describe the system model of the overlay MISO CR system; give the three theorems and relevant demonstrations. The condition of simulation and result are given in Sections III. Finally, conclusion is drawn in Section VI.

The following notations are used in this paper: Matrices and vectors are denoted using boldface upper and lower-case letters, respectively. Superscripts $(\bullet)^{-1},(\bullet)^{T}$, and $(\bullet)^{H}$ stand for the inverse operation, the transpose, and the conjugate transpose, respectively. $\boldsymbol{I}$ and $\mathbf{O}$ represent the identity matrix and zero $m$ atrix, respectively. $\mathbb{C}^{x \times y}$ denotes the space of $(x \times y)$ matrix with co mplex entries. $\mathcal{C N}\left(0, \sigma^{2}\right)$ denotes the complex normal distribution with mean $\mu$ and variance $\sigma^{2}$.

\section{System Model and Problem Formulation}

The system model we are interested in is shown in Fig. 1. The primary network has 3 terminals :one PBS uses 2 antennas and 2 PUs are equipped with a single antenna each. The secondary network consists of one CBS uses 2 ante nnas, 2 CUs with a single antenna each. The response of the pr imary downlink channel, the secondary downlink channel and interference channels between the PBS to CUs and the CBS to PUs are denoted by $\boldsymbol{H}_{p p}, \boldsymbol{H}_{c c}, \boldsymbol{H}_{p c}$, and $\boldsymbol{H}_{c p}$ respectively, where $\boldsymbol{H}_{p p}, \boldsymbol{H}_{c c}, \boldsymbol{H}_{p c}, \boldsymbol{H}_{c p} \in \mathbb{C}^{2 \times 2}$. The CSI of all 
links is assumed to be perfectly known at the CBS, whereas the PBS only knows its own downlink channel. Such assumption can be justified as follows: knowledge of $\boldsymbol{H}_{p p}$ is obtained by extracting the CSI feedback from the PUs to the PBS [12]; the CSI of $\boldsymbol{H}_{c c}$ is estimated at the CUs by decoding the CBS's pilot signals and feeding back it to the CBS; the CSI of $\boldsymbol{H}_{p c}$ is obtained by the CUs by synchronizing to primary's pilot signals in every frame and then feeding back this information to the CBS [15]. $\boldsymbol{H}_{c p}$ is obtained by listening to PU transm ission to the PBS and assuming channel reciprocity due to time-division duplex mode.

The information symbol vector corresponding to PUs can b e denoted as $\boldsymbol{u}_{p}=\left[\begin{array}{ll}u_{p, 1} & u_{c, 2}\end{array}\right]^{T}$ and CUs as $\boldsymbol{u}_{c}=\left[\begin{array}{ll}u_{c, 1} & u_{c, 2}\end{array}\right]^{T}$. $S_{p}$ and $S_{c}$ represent the transmitted symbol vectors from the PBS and CBS after precoding. $P_{p}$ and $P_{c}$ are the total transmitted powers for primary and cognitive signals, respectively. For simplicity, we assume equal power allocation for PUs and CUs. $\quad \boldsymbol{P}_{p p}=\operatorname{diag}\left\{\sqrt{P_{p, 1}} \sqrt{P_{p, 2}}\right\} \quad$ and $\quad \boldsymbol{P}_{c c}=\operatorname{diag}\left\{\sqrt{P_{c, 1}} \sqrt{P_{c, 2}}\right\}$ denote the power loadings for the PUs and CUs signals, respectively. $\tilde{\boldsymbol{u}}_{p}=\boldsymbol{P}_{p p} \boldsymbol{u}_{p}$ and $\tilde{\boldsymbol{u}}_{c}=\boldsymbol{P}_{c c} \boldsymbol{u}_{c}$ [14]are the primar $\mathrm{y}$ and secondary symbol vectors after power loading, respectively.

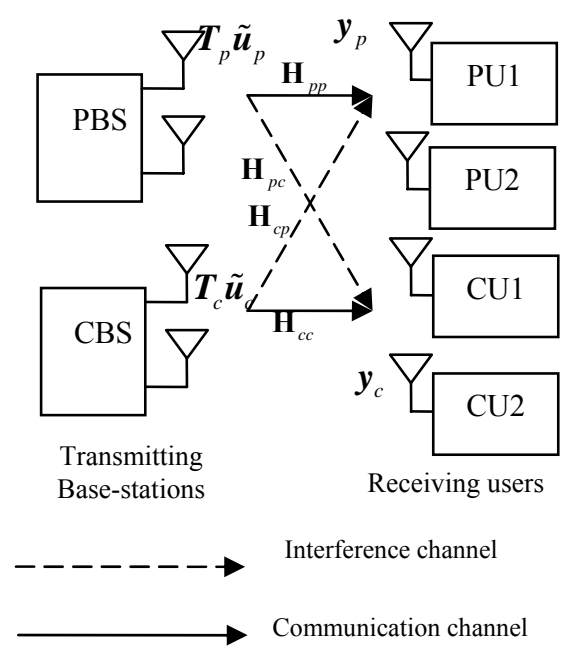

Fig.1. the system model of the CR network

In this paper, we consider linear precoding at the PBS and CBS. $\boldsymbol{T}_{p}$ and $\boldsymbol{T}_{c}$ are precoding matrices at PBS and CBS. $\beta$ is the average transmitted power normalization factor. Thus, the transmitted precoded symbol vectors at the PBS and CBS can be expressed as:

$$
\begin{aligned}
& \boldsymbol{s}_{p}=\frac{\boldsymbol{T}_{p} \tilde{\boldsymbol{u}}_{p}}{\sqrt{\beta}} \\
& \boldsymbol{s}_{c}=\frac{\boldsymbol{T}_{c} \tilde{\boldsymbol{u}}_{c}}{\sqrt{\beta}}
\end{aligned}
$$

The received symbol vectors at the PUs and CUs is given by:

$$
\begin{aligned}
& \boldsymbol{y}_{p}=\boldsymbol{H}_{p p} \boldsymbol{s}_{p}+\boldsymbol{H}_{c p} \boldsymbol{s}_{c}+\boldsymbol{n}_{p} \\
& \boldsymbol{y}_{c}=\boldsymbol{H}_{p c} \boldsymbol{s}_{p}+\boldsymbol{H}_{c c} \boldsymbol{s}_{c}+\boldsymbol{n}_{c}
\end{aligned}
$$

Where $\boldsymbol{n}_{p} \sim \mathcal{C N}\left(0, \sigma_{p}^{2} \sim \boldsymbol{I}\right)$ and $\boldsymbol{n}_{c} \sim \mathcal{C N}\left(0, \sigma_{c}^{2} \sim \boldsymbol{I}\right)$ are the additive white Gaussian noise vectors at the PUs and SUs,.

To simplify the analysis, we let:

$\boldsymbol{H}_{p}=\left[\begin{array}{l}\boldsymbol{H}_{p p} \\ \boldsymbol{H}_{p c}\end{array}\right], \boldsymbol{H}_{c}=\left[\begin{array}{l}\boldsymbol{H}_{c p} \\ \boldsymbol{H}_{c c}\end{array}\right], \boldsymbol{n}=\left[\begin{array}{l}\boldsymbol{n}_{p} \\ \boldsymbol{n}_{c}\end{array}\right], \boldsymbol{y}=\left[\begin{array}{l}\boldsymbol{y}_{p} \\ \boldsymbol{y}_{c}\end{array}\right]$

Thus, (3) and (4) can be rewritten as:

$\boldsymbol{y}=\boldsymbol{H}_{p} \boldsymbol{s}_{p}+\boldsymbol{H}_{c} \boldsymbol{s}_{c}+\boldsymbol{n}$

$=\frac{\boldsymbol{H}_{p} \boldsymbol{T}_{p} \tilde{\boldsymbol{u}}_{p}}{\sqrt{\beta}}+\frac{\boldsymbol{H}_{c} \boldsymbol{T}_{c} \tilde{\boldsymbol{u}}_{c}}{\sqrt{\beta}}+\boldsymbol{n}$

To detect $\tilde{\boldsymbol{u}}_{p}$ and $\tilde{\boldsymbol{u}}_{c}$ from (6), we $\mathrm{n}$ eed to design decoding matrices $\boldsymbol{U}_{p}$ and $\boldsymbol{U}_{c}$ as well as the precoding matrices $\boldsymbol{T}_{p}$ and $\boldsymbol{T}_{c}$, these matrices need satisfy the following theorem.

Theorem 1: The received symbol vectors $\boldsymbol{y}_{p}$ and $\boldsymbol{y}_{c}$ at the PUs and SUs are formulated in (3) and (4), where the transmitted precoded symbol vectors $\boldsymbol{S}_{p}$ and $\boldsymbol{S}_{c}$ at the PBS and CBS are given by (1) and

(2).We can separate $\tilde{\boldsymbol{u}}_{p}$ and $\tilde{\boldsymbol{u}}_{c}$ from (6) if and only if

$$
\boldsymbol{U}_{p} \boldsymbol{H}_{c} \boldsymbol{T}_{c}=\boldsymbol{U}_{c} \boldsymbol{H}_{p} \boldsymbol{T}_{p}=\mathbf{0}
$$

Proof: Necessity is obvious, we only prove the sufficiency. If the precoding matrices $\boldsymbol{T}_{p}$ and $\boldsymbol{T}_{c}$ as well as decoding matrices $\boldsymbol{U}_{p}$ and $\boldsymbol{U}_{c}$ satisfy (7), multiplying $\boldsymbol{U}_{p}$ to (6), we have

$$
\begin{aligned}
& \boldsymbol{U}_{p} \boldsymbol{y}=\frac{\boldsymbol{U}_{p} \boldsymbol{H}_{p} \boldsymbol{T}_{p} \tilde{\boldsymbol{u}}_{p}}{\sqrt{\beta}}+\frac{\boldsymbol{U}_{p} \boldsymbol{H}_{c} \boldsymbol{T}_{c} \tilde{\boldsymbol{u}}_{c}}{\sqrt{\beta}}+\boldsymbol{U}_{p} \boldsymbol{n} \\
& =\frac{\boldsymbol{V}_{p} \tilde{\boldsymbol{u}}_{p}}{\sqrt{\beta}}+\boldsymbol{U}_{p} \boldsymbol{n}
\end{aligned}
$$

where

$$
\boldsymbol{V}_{p}=\boldsymbol{U}_{p} \boldsymbol{H}_{p} \boldsymbol{T}_{p}
$$

Similarly, multiplying $\boldsymbol{U}_{c}$ to (6), we have

$$
\begin{aligned}
& \boldsymbol{U}_{c} \boldsymbol{y}=\frac{\boldsymbol{U}_{c} \boldsymbol{H}_{p} \boldsymbol{T}_{p} \tilde{\boldsymbol{u}}_{p}}{\sqrt{\beta}}+\frac{\boldsymbol{U}_{c} \boldsymbol{H}_{c} \boldsymbol{T}_{c} \tilde{\boldsymbol{u}}_{c}}{\sqrt{\beta}}+\boldsymbol{U}_{c} \boldsymbol{n} \\
& =\frac{\boldsymbol{V}_{c} \tilde{\boldsymbol{u}}_{c}}{\sqrt{\beta}}+\boldsymbol{U}_{c} \boldsymbol{n} \\
& \boldsymbol{V}_{c}=\boldsymbol{U}_{c} \boldsymbol{H}_{c} \boldsymbol{T}_{c}
\end{aligned}
$$

where

Further, by multiplying $\boldsymbol{V}_{p}^{-1}$ and $\boldsymbol{V}_{c}^{-1}$ to (8) and (10), respectively, we get

$$
\begin{gathered}
\boldsymbol{V}_{p}^{-1} \boldsymbol{U}_{p} \boldsymbol{y}=\frac{\boldsymbol{I} \tilde{\boldsymbol{u}}_{p}}{\sqrt{\beta}}+\boldsymbol{V}_{p}^{-1} \boldsymbol{U}_{p} \boldsymbol{n} \\
\boldsymbol{V}_{c}^{-1} \boldsymbol{U}_{c} \boldsymbol{y}=\frac{\boldsymbol{I} \tilde{\boldsymbol{u}}_{c}}{\sqrt{\beta}}+\boldsymbol{V}_{c}^{-1} \boldsymbol{U}_{c} \boldsymbol{n}
\end{gathered}
$$

From Theorem 1, we can obtain some algorithms to construct the precoding matrices $\boldsymbol{T}_{p}$ and $\boldsymbol{T}_{c}$ as well as decoding matrices $\boldsymbol{U}_{p}$ and $\boldsymbol{U}_{c}$. One of the algorithms is as following theorem. 
Theorem 2: If $\boldsymbol{U}_{p}=\left[\begin{array}{ll}\boldsymbol{H}_{c c} & -\boldsymbol{H}_{c p}\end{array}\right], \quad \boldsymbol{U}_{c}=\left[\begin{array}{ll}\boldsymbol{H}_{p c} & -\boldsymbol{H}_{p p}\end{array}\right]$, $\boldsymbol{T}_{p}=\left(\boldsymbol{U}_{p} \boldsymbol{H}_{p}\right)^{-1}, \boldsymbol{T}_{c}=\left(\boldsymbol{U}_{c} \boldsymbol{H}_{c}\right)^{-1}$, and

$\boldsymbol{H}_{c c} \boldsymbol{H}_{c p}=\boldsymbol{H}_{c p} \boldsymbol{H}_{c c}$

$\boldsymbol{H}_{p c} \boldsymbol{H}_{p p}=\boldsymbol{H}_{p p} \boldsymbol{H}_{p c}$

then

$\boldsymbol{U}_{p} \boldsymbol{y}=\frac{\tilde{\boldsymbol{u}}_{p}}{\sqrt{\beta}}+\boldsymbol{U}_{p} \boldsymbol{n}$

$\boldsymbol{U}_{c} \boldsymbol{y}=\frac{\tilde{\boldsymbol{u}}_{c}}{\sqrt{\beta}}+\boldsymbol{U}_{c} \boldsymbol{n}$

Proof: If $\boldsymbol{U}_{p}=\left[\begin{array}{ll}\boldsymbol{H}_{c c} & -\boldsymbol{H}_{c p}\end{array}\right], \boldsymbol{U}_{c}=\left[\begin{array}{ll}\boldsymbol{H}_{p c} & -\boldsymbol{H}_{p p}\end{array}\right]$, due to (7), we have $\boldsymbol{U}_{p} \boldsymbol{H}_{c}=\left[\begin{array}{ll}\boldsymbol{H}_{c} & -\boldsymbol{H}_{q p}\end{array}\right]\left[\begin{array}{l}\boldsymbol{H}_{q} \\ \boldsymbol{H}_{c c}\end{array}\right]=\boldsymbol{H}_{c} \boldsymbol{H}_{q}-\boldsymbol{H}_{q p} \boldsymbol{H}_{c}$

$\boldsymbol{U}_{c} \boldsymbol{H}_{p}=\left[\begin{array}{ll}\boldsymbol{H}_{p c} & -\boldsymbol{H}_{p p}\end{array}\right]\left[\begin{array}{c}\boldsymbol{H}_{p p} \\ \boldsymbol{H}_{p c}\end{array}\right]=\boldsymbol{H}_{p c} \boldsymbol{H}_{p p}-\boldsymbol{H}_{p p} \boldsymbol{H}_{p c}$

Due to (14) and (15), (18) and (19) can be simplified into

$\boldsymbol{U}_{p} \boldsymbol{H}_{c}=\left[\begin{array}{ll}\boldsymbol{H}_{c c} & -\boldsymbol{H}_{c p}\end{array}\right]\left[\begin{array}{l}\boldsymbol{H}_{c p} \\ \boldsymbol{H}_{c c}\end{array}\right]=\mathbf{0}$

$\boldsymbol{U}_{c} \boldsymbol{H}_{p}=\left[\begin{array}{ll}\boldsymbol{H}_{p c} & -\boldsymbol{H}_{p p}\end{array}\right]\left[\begin{array}{l}\boldsymbol{H}_{p p} \\ \boldsymbol{H}_{p c}\end{array}\right]=\mathbf{0}$

Then, (8) and (10) can be changed into:

$$
\begin{aligned}
& \boldsymbol{U}_{p} \boldsymbol{y}=\frac{\boldsymbol{U}_{p} \boldsymbol{H}_{p} \boldsymbol{T}_{p} \tilde{\boldsymbol{u}}_{p}}{\sqrt{\beta}}+\boldsymbol{U}_{p} \boldsymbol{n} \\
& \boldsymbol{U}_{c} \boldsymbol{y}=\frac{\boldsymbol{U}_{c} \boldsymbol{H}_{p} \boldsymbol{T}_{p} \tilde{\boldsymbol{u}}_{p}}{\sqrt{\beta}}+\boldsymbol{U}_{c} \boldsymbol{n}
\end{aligned}
$$

Substitute $\boldsymbol{T}_{p}=\left(\boldsymbol{U}_{p} \boldsymbol{H}_{p}\right)^{-1}$ and $\boldsymbol{T}_{c}=\left(\boldsymbol{U}_{c} \boldsymbol{H}_{c}\right)^{-1}$ into (22) and (23), we will have the results in (16) and (17).

In applications, the channel conditions (14) and (15) of Theorem 2 are very strict. PBS and CBS need to select the PUs and CUs to satisfy the channel conditions to ensure the detections of $\tilde{\boldsymbol{u}}_{p}$ and $\tilde{\boldsymbol{u}}_{c}$ from (6). To solve th e problem, we hav e following theorem to construct $\boldsymbol{T}_{p}, \boldsymbol{T}_{c}, \boldsymbol{U}_{p}$ and $\boldsymbol{U}_{c}$, which can remove the limitations of (14) and (15) of Theorem 2.

Theorem 3: If $\boldsymbol{U}_{p}=\left[\begin{array}{ll}\boldsymbol{H}_{c p}^{-1} & -\boldsymbol{H}_{c c}^{-1}\end{array}\right], \boldsymbol{U}_{c}=\left[\begin{array}{ll}\boldsymbol{H}_{p p}^{-1} & -\boldsymbol{H}_{p c}^{-1}\end{array}\right]$, $\boldsymbol{T}_{p}=\left(\boldsymbol{U}_{p} \boldsymbol{H}_{p}\right)^{-1}, \boldsymbol{T}_{c}=\left(\boldsymbol{U}_{c} \boldsymbol{H}_{c}\right)^{-1}$, then $\tilde{\boldsymbol{u}}_{p}$ and $\tilde{\boldsymbol{u}}_{c}$ can be obtained from (16) and (17).

Proof: If $\boldsymbol{U}_{p}=\left[\begin{array}{ll}\boldsymbol{H}_{c p}^{-1} & -\boldsymbol{H}_{c c}^{-1}\end{array}\right], \boldsymbol{U}_{c}=\left[\begin{array}{ll}\boldsymbol{H}_{p p}^{-1} & -\boldsymbol{H}_{p c}^{-1}\end{array}\right]$, due to

(7), we have

$\boldsymbol{U}_{p} \boldsymbol{H}_{c}=\left[\begin{array}{ll}\boldsymbol{H}_{q}^{-1} & -\boldsymbol{H}_{c}^{-1}\end{array}\right]\left[\begin{array}{l}\boldsymbol{H}_{c p} \\ \boldsymbol{H}_{c c}\end{array}\right]=\boldsymbol{H}_{q}^{-1} \boldsymbol{H}_{c}-\boldsymbol{H}_{c}^{-1} \boldsymbol{H}_{c}$
$\boldsymbol{U}_{c} \boldsymbol{H}_{p}=\left[\begin{array}{ll}\boldsymbol{H}_{p p}^{-1} & -\boldsymbol{H}_{p c}^{-1}\end{array}\right]\left[\begin{array}{l}\boldsymbol{H}_{p p} \\ \boldsymbol{H}_{p c}\end{array}\right]=\boldsymbol{H}_{p p}^{-1} \boldsymbol{H}_{p p}-\boldsymbol{H}_{p c}^{-1} \boldsymbol{H}_{p c}$

$=\boldsymbol{I}-\boldsymbol{I}=\mathbf{0}$

From Theorem 1, (8) and (10) are changed into (22) and (23).

Substitute $\boldsymbol{U}_{p}=\left[\begin{array}{ll}\boldsymbol{H}_{c p}^{-1} & -\boldsymbol{H}_{c c}^{-1}\end{array}\right]$ and $\boldsymbol{U}_{c}=\left[\begin{array}{ll}\boldsymbol{H}_{p p}^{-1} & -\boldsymbol{H}_{p c}^{-1}\end{array}\right]$ into $\boldsymbol{T}_{p}=\left(\boldsymbol{U}_{p} \boldsymbol{H}_{p}\right)^{-1}$ and $\boldsymbol{T}_{c}=\left(\boldsymbol{U}_{c} \boldsymbol{H}_{c}\right)^{-1}$, we can obtain $\boldsymbol{T}_{p}$ and $\boldsymbol{T}_{c}$, then from (22) and (23), we will have the results in (16) and (17).

\section{Simulation Results}

From Theorem 3, we can get th e decoding matrices $\boldsymbol{U}_{p}=\left[\begin{array}{ll}\boldsymbol{H}_{c p}^{-1} & -\boldsymbol{H}_{c c}^{-1}\end{array}\right]$ and $\boldsymbol{U}_{c}=\left[\begin{array}{ll}\boldsymbol{H}_{p p}^{-1} & -\boldsymbol{H}_{p c}^{-1}\end{array}\right]$, precoding matrices $\boldsymbol{T}_{p}=\left(\boldsymbol{U}_{p} \boldsymbol{H}_{p}\right)^{-1}$ and $\boldsymbol{T}_{c}=\left(\boldsymbol{U}_{c} \boldsymbol{H}_{c}\right)^{-1}$.

The decoding results are obtained from (16) and (17),

$$
\begin{aligned}
& \boldsymbol{U}_{p} \boldsymbol{y}=\frac{\tilde{\boldsymbol{u}}_{p}}{\sqrt{\beta}}+\boldsymbol{U}_{p} \boldsymbol{n}, \\
& \boldsymbol{U}_{c} \boldsymbol{y}=\frac{\tilde{\boldsymbol{u}}_{c}}{\sqrt{\beta}}+\boldsymbol{U}_{c} \boldsymbol{n},
\end{aligned}
$$

where $\beta$ is the average transmitted power normalization factor.

According to theorem 3 , we de sign the the precoding and decoding matrices in the simulations. Then, we analysize the BER performance of PBS and CBS in the s ystem model, capacity of the primary user and the cognitive user channel are given too.

\subsection{BER Simulation}

Conditions of simulation experiment: BPSK modulation, Rayleigh flat-fading channel, code rate $0.75,10000$ data frames per SNR point.

Assume the cognitive downlink cha nnel $\boldsymbol{H}_{c c}$ is $\boldsymbol{H}_{c c}=\left[\begin{array}{ll}0.95 & 0.21 \\ 0.37 & 0.85\end{array}\right]$,it is estimated at the CUs b y decoding the CBS's pilot signals and feeding back the estimate of CSI to the CBS. The knowledge of $\boldsymbol{H}_{p c}$ is obtained at the CUs b y synchronizing to primary's pilot signals in every frame and then feeding back this information to the CBS. And le t $\boldsymbol{H}_{p c}=\left[\begin{array}{cc}0.4 & 0.38 \\ 0.21 & 0.54\end{array}\right] . \boldsymbol{H}_{c p}$ It is obtained by listening to PU tran smission to the PBS and assu ming channel reciprocity due to time-division duplex mode. Knowledge of $\boldsymbol{H}_{p p}$ is obtained by extracting the CSI feedback from PUs to PBS.

Each of them are $\boldsymbol{H}_{c p}=\left[\begin{array}{ll}0.62 & 0.31 \\ 0.24 & 0.53\end{array}\right]$ and $\boldsymbol{H}_{p p}=\left[\begin{array}{cc}0.9 & 0.3 \\ 0.4 & 0.73\end{array}\right]$.

$=\boldsymbol{I}-\boldsymbol{I}=\mathbf{0}$ 


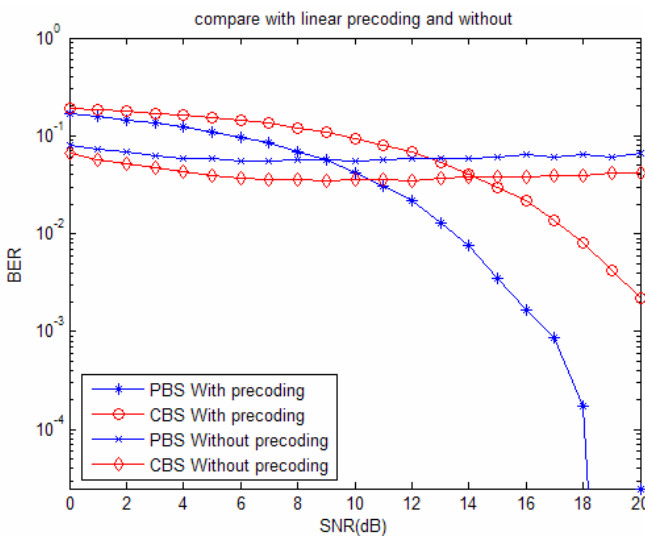

Fig. 3 the BER performance of the PBS and CBS with linear precoding and without

From the Fig.3, we can s ee that with the increase of the S NR, the BER performance of the PBS and CBS with linear precoding is decrease significantly.

\subsection{Channel Capacity}

We compute the capacity by utilizing a pre-whitened channel matrix in order to make the interference-plus-noise appear white. Apply the proposed scheme, due to Theorem 2, there will be no interference for the sharing channel of the PBS and CBS. Thus, the capacity for PBS and CBS can be computed by $\boldsymbol{H}_{p p}$ and $\boldsymbol{H}_{c c}$,

$$
\begin{aligned}
& C_{p p}=\log _{2}\left(\operatorname{det}\left(I+S N R \cdot \boldsymbol{H}_{p p} \boldsymbol{H}_{p p}^{T}\right)\right) \\
& C_{c c}=\log _{2}\left(\operatorname{det}\left(I+S N R \cdot \boldsymbol{H}_{c c} \boldsymbol{H}_{c c}^{T}\right)\right)
\end{aligned}
$$

The following results of Fig. 4 and Fig.5 are based on the calculations of (26) and (27).

Conditions of simulation experiment: Using the Monte Carlo method, the simulation results are the average of 10,000 channels.

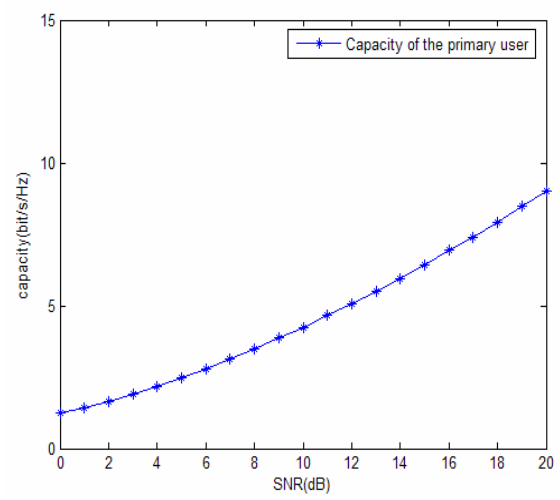

Fig.4 the capacity of the primary user channel

Though the BER performance of the primary user and the cognitive user with linear precoding is different. The cap acity of the prim ary user channel $\boldsymbol{H}_{p p}$ and the cognitive channel $\boldsymbol{H}_{c c}$ are very close.

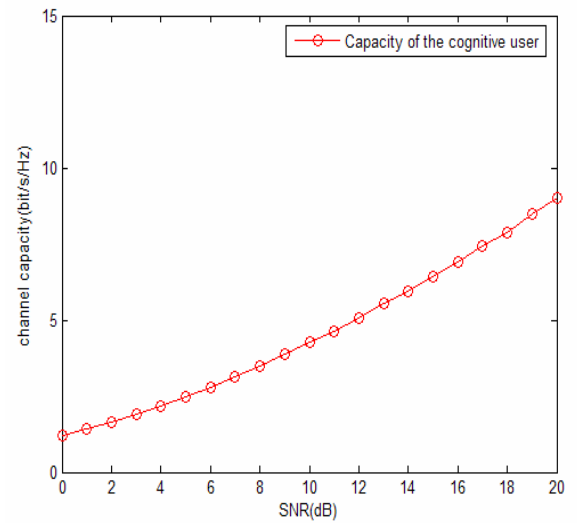

Fig. 5 the capacity of the cognitive user channel

\section{Conclusions}

In this paper, we have considered spectrum sharing in a CR MISO downlink network, where the PBS and CBS transmit to a number of PUs and SUs, respectively. We have proposed the linear precoding and $\mathrm{d}$ ecoding to exploit the interferences the CBS to the PBS and given the method to design the linear precoding and decoding matrices. The simulation result shows the efficiency of the proposed scheme.

\section{References}

[1] Federal Communications Commission, "Spectrum policy task for ce," Rep. ET Docket No. 02-135, Nov. 2002.

[2] Y. Xiao, Y. K. Zhang, Qu Guangzhi, Kim Kiseon, "Spatial multiplexing algorithms of cognitive base-stations", IET 3rd International Conference on Wireless, Mobile and Multimedia Networks (ICWMNN 2010), 2010, pp.221-224.

[3] Y. L. An, Y. Xiao, "Multi-users cooperative transmitting algorithm in cognitive WLAN", Journal of Networks, 2012, 7(8):1164-1169.

[4] A. Goldsmith, S.A. Jafar, I. Maric, and S. Srinivasa, "Breaking spectrum gridlock with cognitive radios: An information theoretic perspective," Proc.IEEE, vol. 97, no. 5, pp. 894-914, May 2009.

[5] R. Zhang, Y.-C. Liang, and S. Cui, "Dynamic resource allocation in cognitive radio networks," IEEE Signal Processing Magazine, vol. 27,no 3, pp. 102-114, May 2010.

[6] N. Devroye, P. M itran, and V. Tarokh, "Achievable $r$ ates in cognitiveradio channels,” IEEE Trans. Inf. Theo ry, vol. 52, no. 5, pp. 1813-1827,May 2006.

[7] A. Jovicic and P. Viswanath, "Cognitive radio: An information theoretic perspective," IEEE Trans. Inf. Theory, vol. 55, no. 9, pp. 39453958,Sep. 2009.

[8] J. Jiang and Y. Xin, "On the achie vable rate regions for interference channels with degr aded message sets," IEEE Trans. Inf. Theory, vol. 54,no. 10, pp. 4707-4712, Oct. 2008.

[9] S. H. Seyedmehdi, Y. Xin, and Y. Lian, "An achievable rate region for the causal cognitive radio," in P roc. 45th Annu. Allerton Conf. Commun.,Control Comput., Monticello, IL, Sep. 2007, pp. 783-790.

[10] Y. Han, A. Pandharipande, and S. Ting, "Cooperative decode-andforward relaying for secondary spectrum access," IEEE Transactions on Wireless Communications, vol. 8, no. 10, pp. 4945-4950, Oct. 2009.

[11] Y. Li, P. Wang, and D. Niyato, "Optimal power allocation for secondary users in cogniti ve relay networks," in $\operatorname{Pr}$ oc. IEEE Wireless Communications and Networking Conf. (WCNC), Mar. 2011, pp. 862867.

[12] C. Masouros and T. Ratna rajah, "Utilization of pri mary-secondary crossinterference via adaptive prec oding in c ognitive relay assisted 
MIMO wireless systems," in Proc. IEEE ICC, Kyoto, Japan, Jun. 5-9, 2011,pp. 1-5.

[13] C. Masouros and T. Ratnarajah, "Interference as a source of green signal power in cognitive rela $\mathrm{y}$ assisted co-existing MIM O wireless transmissions,"IEEE Trans.Commun., vol. 60, no. 2, pp. 525-536, Feb. 2012.

[14] A. Jovicic and P. Viswanath, "Cognitive radio: An information theoretic perspective," IEEE Trans. Inf. Theory, vol. 55, no. 9, pp. 39453958,Sep. 2009.

[15] F. Negro, I. Ghauri, and D. T. M. Slock, "Transmission techniques and channel estimation for spatial interweave $\mathrm{T}$ DD cognitive radio systems,"in Proc. 43rd Asilomar Conf. Signals Sy st. Comput., Pacific Grove, CA,Nov. 1-4, 2009, pp. 523-527.

[16] Y. L. An, Y. Xiao, G. Qu, "Multi-source cooperative MIMO cognitive network based on distributed interference alignment", 4th IE T International Conference on Wireless, Mobile \& Multimedia Networks (ICWMMN 2011), 2011, pp.160-164.

[17] Y. F. Li, Y. Xiao, M. H. Lee, "MIMO interference alignment for cognitive radio network, 4th IET International Conference on Wireless", Mobile \& Multimedia Networks (ICWMMN 2011), 2011, pp.127-130.

[18] Y. Y. Li, Y. Xiao, M. H. L ee, "Decoding algo rithm and BE R performance of interference alignment and cancellation system in MIMO networks", 4th I ET International Conference on W ireless", Mobile \& Multimedia Networks (ICWMMN 2011), 2011, pp.131-136.

\section{Creative Commons Attribution License 4.0 (Attribution 4.0 International, CC BY 4.0)}

This article is published under the terms of the Creative Commons Attribution License 4.0

https://creativecommons.org/licenses/by/4.0/deed.en_US 